\title{
STUDY OF SEISMIC BEHAVIOR OF CONVENTIONAL AND RC WALL BUILDING
}

\author{
Pradeep B ${ }^{1}$, Shiva Kumar KS ${ }^{2}$, Ambrish $\mathbf{G}^{3}$ \\ ${ }^{1}$ Post Graduate Student, Department of Civil Engineering, GAT, Bengaluru, Karnataka, India \\ ${ }^{2}$ Assistant Professor, Department of Civil Engineering, GAT, Bengaluru, Karnataka, India \\ ${ }^{3}$ Executive Director, Executive Director Concons Consultants Pvt. Ltd., Bengaluru, Karnataka, India
}

\begin{abstract}
In multi storey buildings earthquake forces will have a very high impact on any form of the structure. In recent years the construction industry had developed many new technologies. MIVAN construction technology is also one of them. It has been adopted all over the world because the construction is speedy. This work is conducted to come up with the realistic conventional and RC wall building models to study the seismic behavior under fixed base. The main objective of the work is to study the seismic behavior of the conventional and $R C$ wall building and to study the performance of both the buildings under earthquake generated forces. In this work a G+7 storey conventional (beam, column, slab) building and RC Wall Building (mivan construction) of same plan is considered. The models of both buildings are created using ETABS version 15.2.0. To study the seismic behavior of the both conventional and RC Wall buildings the response spectrum analysis was performed on both models as per the Indian Standard Code IS 1893:2002 Part1. From the analysis results it was found that the RC Wall Building performs better compared to the Conventional Building.
\end{abstract}

Keywords: Mivan Construction, RC Wall Building, Conventional Building, ETABS Version 15.2.0.

\section{INTRODUCTION}

In recent years the development in urbanization is showing rapid growth all over the world. This has initiated the concept of multi-storey buildings. The construction technology is improving day by day, one of such recent construction technologies is mivan construction technology. The conventional buildings are the ones which are built with the conventional construction practice that is beam-column and slab concept. In the recent past mivan construction technology has developed and widely used all around the world and even in India this technology has been adopted in may major cities. The buildings built with this technology consist of the RC wall with the slab resting on them. This technology is widely adopted because of the speedy construction. These multi-storey buildings are highly susceptible to the lateral forces induced by the strong earthquake ground motion.

\section{OBJECTIVE OF THE WORK}

The main objective of this work is to study the seismic behavior of the RC Wall Building in comparison with the conventional one. For this, in the present work the behaviors of the buildings are compared in all the four earthquake zones as per the Indian Standard Code IS 1893 : 2002.

\section{METHEDOLOGY}

In the present project work two different types of reinforced concrete buildings with $\mathrm{G}+7$ storeys are being considered to study the seismic responses in fixed condition. The two different models considered are beam-column, slab type conventional $\mathrm{RC}$ building and the other one is $\mathrm{RC}$ wall type building. Both the type of the buildings were modeled using the software package ETABS version 15.2.0. The full description of both models generated in ETABS with figures is as given.

\begin{tabular}{|l|l|}
\hline Conventional Building & \\
\hline Number of floors & $\mathrm{G}+7$ \\
\hline Height of each floor & $3.15 \mathrm{~m}$ \\
\hline Size of the column & $600 \mathrm{~mm}$ X 200 \\
\hline Size of the beam & $200 \mathrm{~mm}$ X 450 \\
\hline Thickness of the slab & $150 \mathrm{~mm}$ \\
\hline Thickness of the concrete block & $200 \mathrm{~mm}$ \\
\hline Thickness of the concrete block & $150 \mathrm{~mm}$ \\
\hline Height of the plinth above the & $1.5 \mathrm{~m}$ \\
\hline Height of the parapet & $1 \mathrm{~m}$ \\
\hline Thickness of the parapet wall & $150 \mathrm{~mm}$ \\
\hline Number of lift cores & 2 \\
\hline Size of the lift core & $2.05 \mathrm{~m} \mathrm{X} \mathrm{2.15} \mathrm{m}$ \\
\hline Thickness of the lift core wall & $200 \mathrm{~mm}$ \\
\hline RC Wall Building & \\
\hline Number of floors & $\mathrm{G}+7$ \\
\hline Height of each floor & $3.15 \mathrm{~m}$ \\
\hline Thickness of the RC Wall & $160 \mathrm{~mm}$ \\
\hline Thickness of the slab & $150 \mathrm{~mm}$ \\
\hline Height above the foundation & $1.5 \mathrm{~m}$ \\
\hline Number of lift cores & 2 \\
\hline Size of the lift core & $2.05 \mathrm{~m} \mathrm{X} \mathrm{2.15} \mathrm{m}$ \\
\hline
\end{tabular}




\begin{tabular}{|l|l|}
\hline Thickness of the lift core wall & $200 \mathrm{~mm}$ \\
\hline Material Properties & $\mathrm{M} 25$ \\
\hline Grade of concrete & $\mathrm{Fe} 415$ \\
\hline Grade of reinforcing steel & $17.65 \mathrm{kN} / \mathrm{m}^{3}$ \\
\hline Density of the concrete blocks & $1.5 \mathrm{kN} / \mathrm{m}^{2}$ \\
\hline Loading & $2 \mathrm{kN} / \mathrm{m}^{2}$ \\
\hline Floor finish & $3 \mathrm{kN} / \mathrm{m}^{2}$ \\
\hline Load on all rooms & $2 \mathrm{kN} / \mathrm{m}^{2}$ \\
\hline Load on Staircases &
\end{tabular}

All the loads applied are as per Indian Standard Code IS875:1987 Part 1 and Part 2. The Study was conducted considering all the four earthquake zones as per the Code IS1893:2002 Part 1.Both types of the building are studied in earthquake zones Zone II, Zone III, Zone IV and Zone V considering the medium soil that is soil type II.

The design horizontal seismic coefficient Ah for a structure shall be determined by the following expression:

$$
A_{h}=\frac{Z I S_{a}}{2 R g}
$$

The total design lateral force or design seismic base shear (Vb) along any principal direction shall be determined by the following expression:

$$
V_{B}=A_{h} W
$$

\section{RESULTS}

From the analysis results some of the parameters such as Displacements, Drifts and Base Shear are compared for the Conventional Building and RC Wall Building. The analysis results, tabulated and represented in the form of plots are given below.

Table-1: Storey Displacement, Conventional v/s RC Wall Building, Zone II (X-direction)

Fig-1: Plan of the building

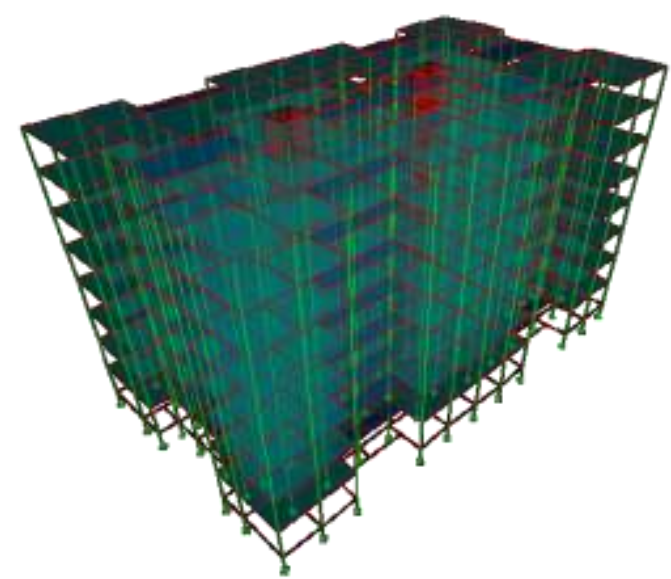

Fig-2: Conventional Building

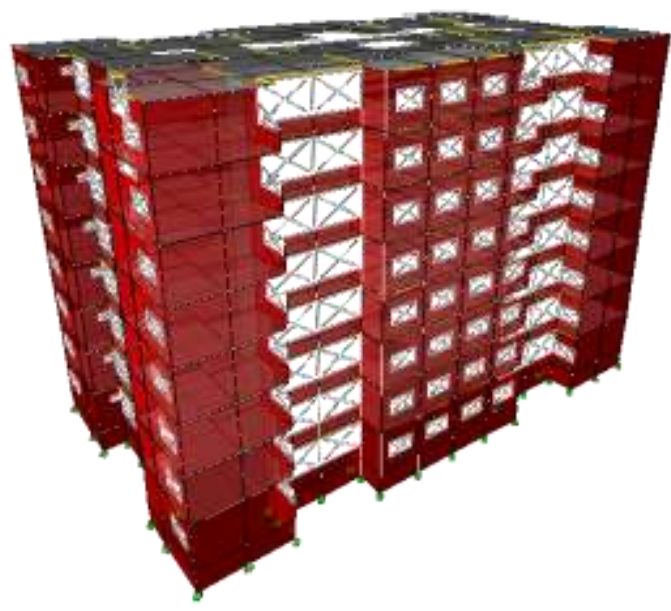

Fig-3: RC Wall Building

\begin{tabular}{|l|l|}
\hline \multicolumn{2}{|l|}{ Storey Displacement, Zone II (X-direction) } \\
\hline $\begin{array}{l}\text { Conventional Building, Zone } \\
\text { II }\end{array}$ & $\begin{array}{l}\text { RC wall Building, Zone } \\
\text { II }\end{array}$ \\
\hline 0 & 0 \\
\hline 6.37 & 0.0404 \\
\hline 13.50 & 0.0714 \\
\hline 21.02 & 0.1023 \\
\hline 28.27 & 0.1317 \\
\hline 34.87 & 0.1582 \\
\hline 40.58 & 0.1809 \\
\hline 45.27 & 0.1991 \\
\hline 50.00 & 0.2168 \\
\hline
\end{tabular}

Table-2: Storey Displacement, Conventional v/s RC Wall Building, Zone III (X-direction)

\begin{tabular}{|l|l|}
\hline \multicolumn{2}{|l|}{ Storey Displacement, Zone III (X-direction) } \\
\hline $\begin{array}{l}\text { Conventional Building, Zone } \\
\text { III }\end{array}$ & $\begin{array}{l}\text { RC wall Building, Zone } \\
\text { III }\end{array}$ \\
\hline 0 & 0 \\
\hline 10.20 & 0.0647 \\
\hline 21.61 & 0.1142 \\
\hline 33.63 & 0.1637 \\
\hline 45.23 & 0.2106 \\
\hline 55.79 & 0.2531 \\
\hline 64.93 & 0.2895 \\
\hline 72.43 & 0.3185 \\
\hline 78.40 & 0.3468 \\
\hline
\end{tabular}


Table-3: Storey Displacement, Conventional v/s RC Wall Building, Zone IV (X-direction)

\begin{tabular}{|l|l|}
\hline \multicolumn{2}{|l|}{ Storey Displacement, Zone IV ( X-direction) } \\
\hline $\begin{array}{l}\text { Conventional Building, } \\
\text { Zone IV }\end{array}$ & $\begin{array}{l}\text { RC wall Building, Zone } \\
\text { IV }\end{array}$ \\
\hline 0 & 0 \\
\hline 15.30 & 0.0971 \\
\hline 32.41 & 0.1713 \\
\hline 50.45 & 0.2455 \\
\hline 67.85 & 0.3160 \\
\hline 83.69 & 0.3797 \\
\hline 97.40 & 0.4342 \\
\hline 108.66 & 0.4778 \\
\hline 117.61 & 0.5203 \\
\hline
\end{tabular}

Table-4: Storey Displacement, Conventional v/s RC Wall Building, Zone V (X-direction)

\begin{tabular}{|l|l|}
\hline \multicolumn{2}{|l|}{ Storey Displacement, Zone V (X-direction) } \\
\hline $\begin{array}{l}\text { Conventional Building, } \\
\text { Zone V }\end{array}$ & $\begin{array}{l}\text { RC wall Building, Zone } \\
\text { V }\end{array}$ \\
\hline 0 & 0 \\
\hline 22.95 & 0.1456 \\
\hline 48.61 & 0.2569 \\
\hline 75.67 & 0.3683 \\
\hline 101.78 & 0.4739 \\
\hline 125.54 & 0.5695 \\
\hline 146.10 & 0.6513 \\
\hline 162.98 & 0.7167 \\
\hline 176.41 & 0.7804 \\
\hline
\end{tabular}

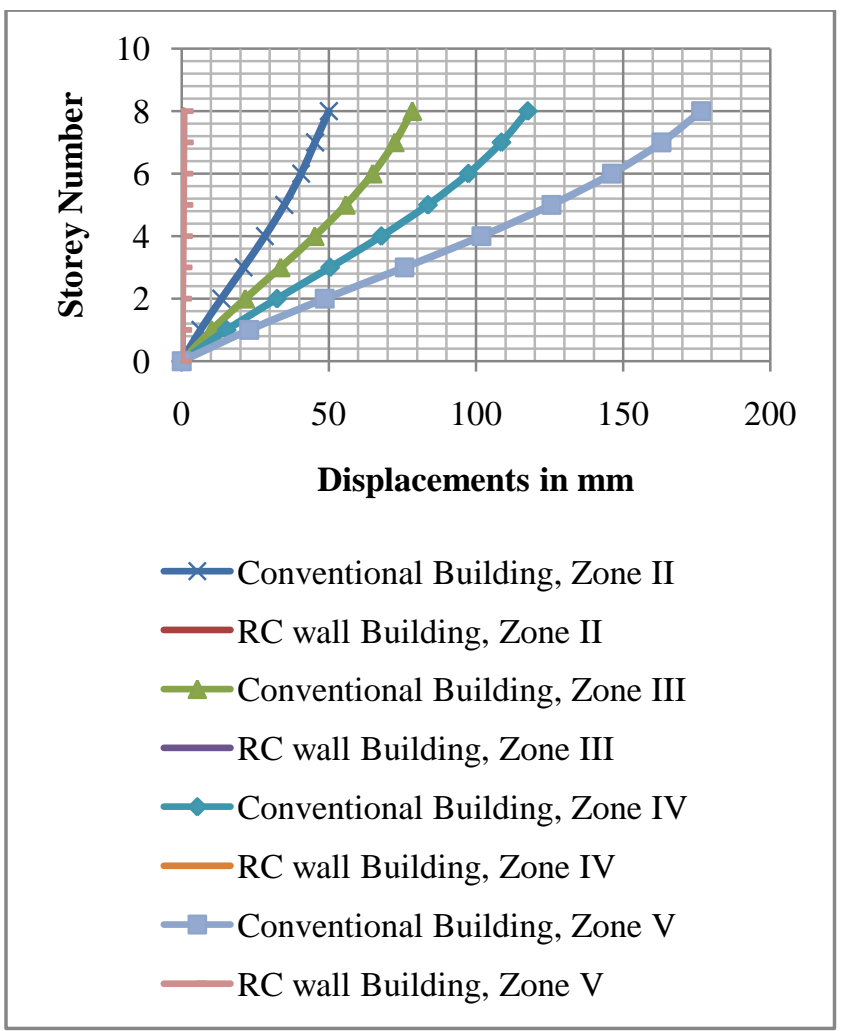

Fig-4: Storey Displacement, Conventional v/s RC Wall Building, Zone II, III, IV, V (X-direction)
Table-5: Storey Displacement, Conventional v/s RC Wall Building, Zone II (Y-direction)

Storey Displacement, Zone II (Y-direction)

Conventional Building $\quad$ RC wall Building

\begin{tabular}{|l|l|}
\hline 0 & 0 \\
\hline 3.48 & 0.0655 \\
\hline 7.72 & 0.1281 \\
\hline 12.31 & 0.1981 \\
\hline 16.76 & 0.2711 \\
\hline 20.80 & 0.3435 \\
\hline 24.26 & 0.4125 \\
\hline 27.09 & 0.4760 \\
\hline 29.34 & 0.5443 \\
\hline
\end{tabular}

Table-6: Storey Displacement, Conventional v/s RC Wall Building, Zone III (Y-direction)

\begin{tabular}{|l|l|}
\hline Storey Displacement, Zone III (Y-direction) \\
\hline Conventional Building & RC wall Building \\
\hline 0 & 0 \\
\hline 5.57 & 0.1047 \\
\hline 12.35 & 0.2049 \\
\hline 19.70 & 0.3168 \\
\hline 26.82 & 0.4336 \\
\hline 33.28 & 0.5495 \\
\hline 38.82 & 0.6599 \\
\hline 43.34 & 0.7615 \\
\hline 46.95 & 0.8707 \\
\hline
\end{tabular}

Table-7: Storey Displacement, Conventional v/s RC Wall Building, Zone IV (Y-direction)

\begin{tabular}{|l|l|}
\hline \multicolumn{2}{|l|}{ Storey Displacement, Zone IV (Y-direction) } \\
\hline Conventional Building & RC wall Building \\
\hline 0 & 0 \\
\hline 8.36 & 0.1571 \\
\hline 18.52 & 0.3074 \\
\hline 29.54 & 0.4752 \\
\hline 40.23 & 0.6504 \\
\hline 49.93 & 0.8243 \\
\hline 58.24 & 0.9900 \\
\hline 65.01 & 1.1423 \\
\hline 70.43 & 1.3061 \\
\hline
\end{tabular}

Table-8: Storey Displacement, Conventional v/s RC Wall Building, Zone V (Y-direction)

\begin{tabular}{|l|l|}
\hline Storey Displacement, Zone V (Y-direction) \\
\hline Conventional Building & RC wall Building \\
\hline 0 & 0 \\
\hline 12.54 & 0.2357 \\
\hline 27.78 & 0.4611 \\
\hline 44.31 & 0.7128 \\
\hline 60.34 & 0.9756 \\
\hline 74.89 & 1.2364 \\
\hline 87.36 & 1.4849 \\
\hline 97.52 & 1.7134 \\
\hline 105.65 & 1.9595 \\
\hline
\end{tabular}




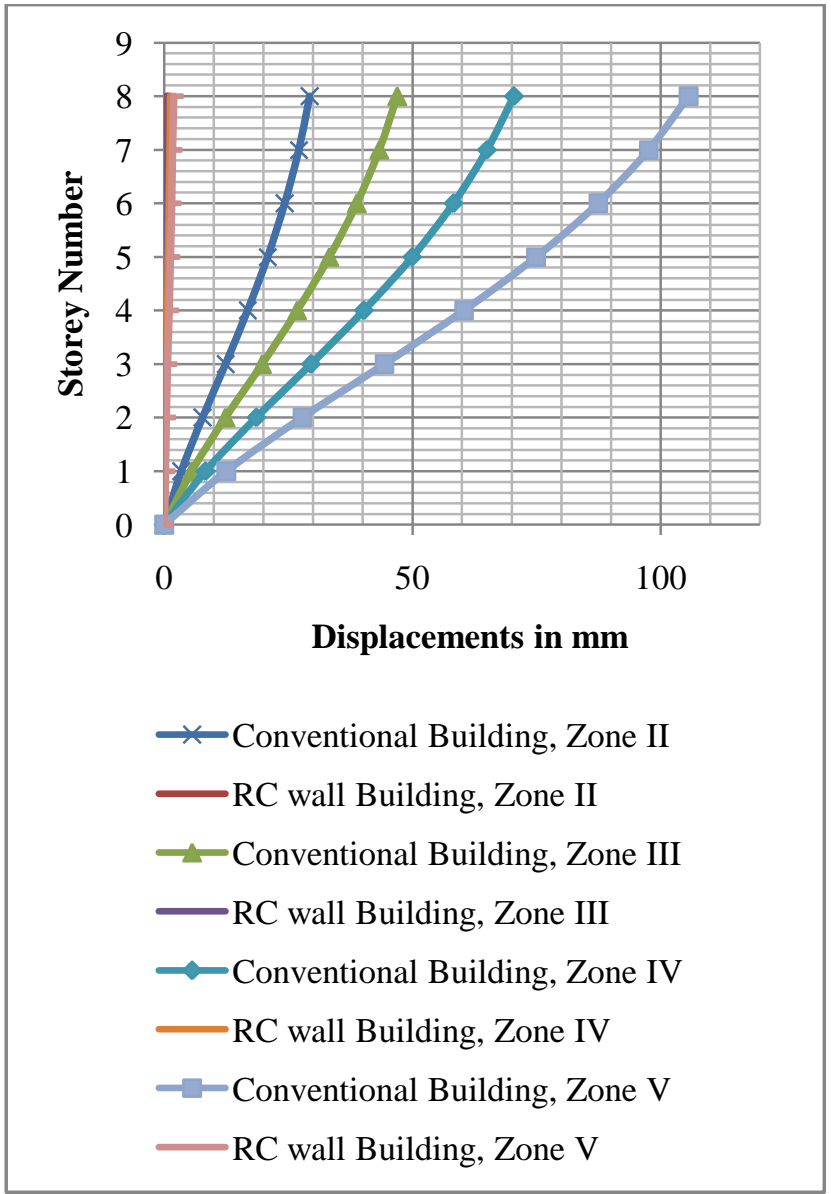

Fig-5: Storey Displacement, Conventional v/s RC Wall Building, Zone II, III, IV, V (Y-direction)

From the above tables and the graphs it can be seen that the RC wall type of building has produced much lesser displacements than the conventional building. This shows that the RC wall type of building offers more resistance to the lateral forces such as seismic forces than the conventional building.

Table-9: Storey Drift, Conventional v/s RC Wall Building, Zone II (X-direction)

\begin{tabular}{|l|l|}
\hline \multicolumn{2}{|l|}{ Storey Drift, Zone II (X-direction) } \\
\hline $\begin{array}{l}\text { Conventional Building, Zone } \\
\text { II }\end{array}$ & $\begin{array}{l}\text { RC wall Building, Zone } \\
\text { II }\end{array}$ \\
\hline 0 & 0 \\
\hline 0.00179 & 0.000010 \\
\hline 0.00227 & 0.000010 \\
\hline 0.00241 & 0.000010 \\
\hline 0.00236 & 0.000009 \\
\hline 0.00219 & 0.000008 \\
\hline 0.00194 & 0.000007 \\
\hline 0.00162 & 0.000006 \\
\hline 0.00128 & 0.000006 \\
\hline
\end{tabular}

Table-10: Storey Drift, Conventional v/s RC Wall Building, Zone III (X-direction)

\begin{tabular}{|l|l|}
\hline \multicolumn{2}{|l|}{ Storey Drift, Zone III (X-direction) } \\
\hline $\begin{array}{l}\text { Conventional Building, Zone } \\
\text { III }\end{array}$ & $\begin{array}{l}\text { RC wall Building, Zone } \\
\text { III }\end{array}$ \\
\hline 0 & 0 \\
\hline 0.00286 & 0.000016 \\
\hline 0.00363 & 0.000016 \\
\hline 0.00386 & 0.000016 \\
\hline 0.00378 & 0.000015 \\
\hline 0.00351 & 0.000014 \\
\hline 0.00310 & 0.000012 \\
\hline 0.00256 & 0.000009 \\
\hline 0.00205 & 0.000009 \\
\hline
\end{tabular}

Table-11: Storey Drift, Conventional v/s RC Wall Building, Zone IV (X-direction)

\begin{tabular}{|l|l|}
\hline \multicolumn{2}{|l|}{ Storey Drift, Zone IV (X-direction) } \\
\hline $\begin{array}{l}\text { Conventional Building, Zone } \\
\text { IV }\end{array}$ & $\begin{array}{l}\text { RC wall Building, Zone } \\
\text { IV }\end{array}$ \\
\hline 0 & 0 \\
\hline 0.00429 & 0.000024 \\
\hline 0.00545 & 0.000024 \\
\hline 0.00579 & 0.000024 \\
\hline 0.00566 & 0.000022 \\
\hline 0.00526 & 0.000020 \\
\hline 0.00465 & 0.000017 \\
\hline 0.00388 & 0.000014 \\
\hline 0.00307 & 0.000014 \\
\hline
\end{tabular}

Table-12: Storey Drift, Conventional v/s RC Wall Building, Zone V (X-direction)

\begin{tabular}{|l|l|}
\hline \multicolumn{2}{|c|}{ Zone V (X-direction) } \\
\hline $\begin{array}{l}\text { Storey Drift, Zone V (X-direction) } \\
\text { V }\end{array}$ & $\begin{array}{l}\text { RC wall Building, Zone } \\
\text { V }\end{array}$ \\
\hline 0 & 0 \\
\hline 0.00644 & 0.000036 \\
\hline 0.00817 & 0.000035 \\
\hline 0.00868 & 0.000035 \\
\hline 0.00850 & 0.000034 \\
\hline 0.00789 & 0.000030 \\
\hline 0.00698 & 0.000026 \\
\hline 0.00582 & 0.000021 \\
\hline 0.00461 & 0.000021 \\
\hline
\end{tabular}




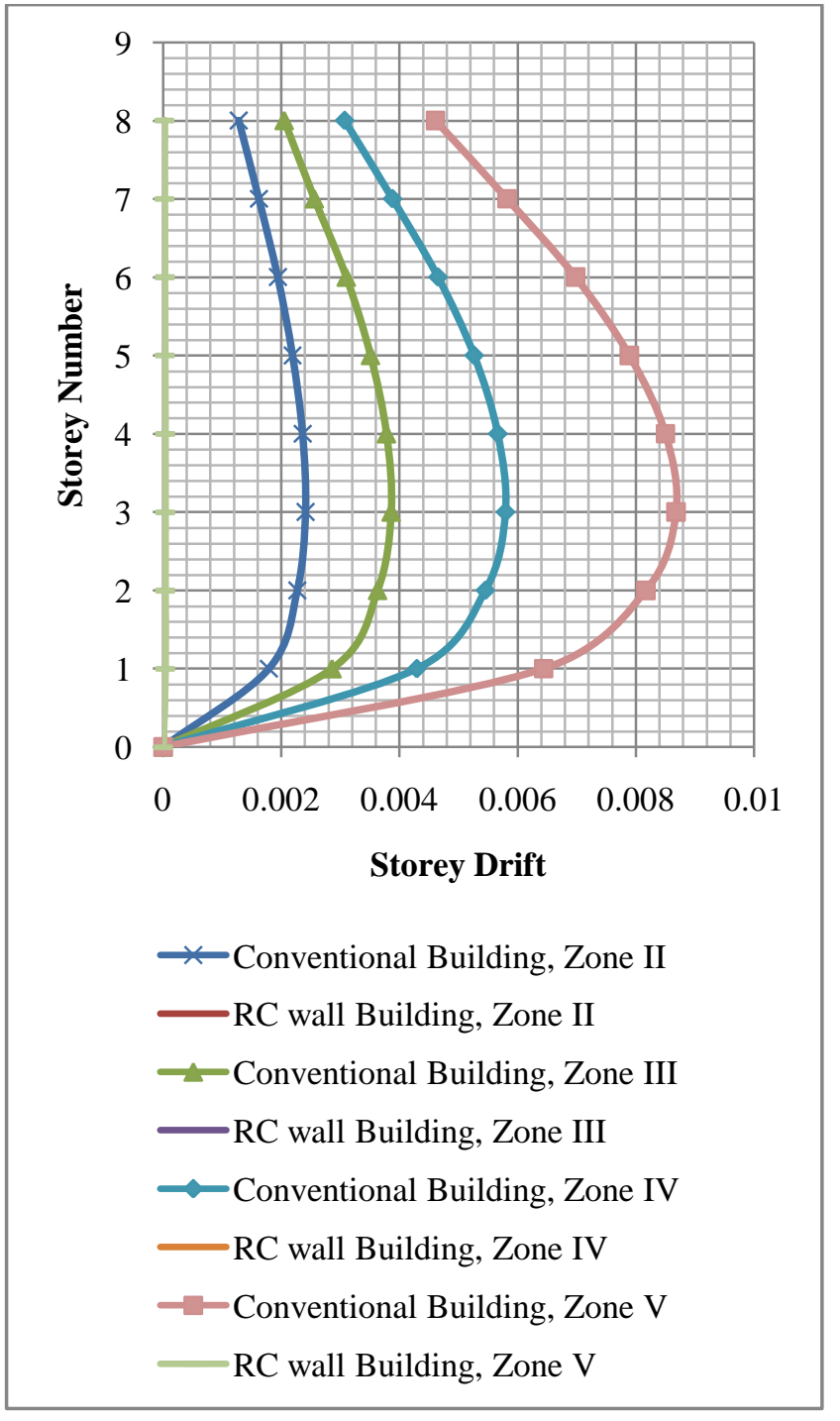

Fig-6: Storey Drift, Conventional v/s RC Wall Building, Zone II, III, IV, V (X-direction)

Table-13: Storey Drift, Conventional v/s RC Wall Building, Zone II (Y-direction)

\begin{tabular}{|l|l|}
\hline \multicolumn{2}{|l|}{ Storey Drift, Zone II (Y-direction) } \\
\hline Conventional Building & RC wall Building \\
\hline 0 & 0 \\
\hline 0.00097 & 0.000017 \\
\hline 0.00135 & 0.000020 \\
\hline 0.00147 & 0.000022 \\
\hline 0.00144 & 0.000023 \\
\hline 0.00132 & 0.000023 \\
\hline 0.00116 & 0.000022 \\
\hline 0.00096 & 0.000020 \\
\hline 0.00077 & 0.000022 \\
\hline
\end{tabular}

Table-14: Storey Drift, Conventional v/s RC Wall Building, Zone III (Y-direction)

\begin{tabular}{|l|l|}
\hline \multicolumn{2}{|l|}{ Storey Drift, Zone III (Y-direction) } \\
\hline Conventional Building & RC wall Building \\
\hline 0 & 0 \\
\hline 0.00155 & 0.000028 \\
\hline 0.00216 & 0.000032 \\
\hline 0.00235 & 0.000036 \\
\hline 0.00230 & 0.000037 \\
\hline 0.00212 & 0.000037 \\
\hline 0.00185 & 0.000035 \\
\hline 0.00153 & 0.000032 \\
\hline 0.00123 & 0.000035 \\
\hline
\end{tabular}

Table-15: Storey Drift, Conventional v/s RC Wall Building, Zone IV (Y-direction)

\begin{tabular}{|l|l|}
\hline \multicolumn{2}{|l|}{ Storey Drift, Zone IV (Y-direction) } \\
\hline Conventional Building & RC wall Building \\
\hline 0 & 0 \\
\hline 0.00233 & 0.000042 \\
\hline 0.00323 & 0.000048 \\
\hline 0.00352 & 0.000053 \\
\hline 0.00345 & 0.000056 \\
\hline 0.00318 & 0.000055 \\
\hline 0.00278 & 0.000053 \\
\hline 0.00230 & 0.000049 \\
\hline 0.00184 & 0.000053 \\
\hline
\end{tabular}

Table-16: Storey Drift, Conventional v/s RC Wall Building, Zone V (Y-direction)

\begin{tabular}{|l|l|}
\hline \multicolumn{2}{|l|}{ Storey Drift, Zone V (Y-direction) } \\
\hline Conventional Building & RC wall Building \\
\hline 0 & 0 \\
\hline 0.00349 & 0.000063 \\
\hline 0.00485 & 0.000072 \\
\hline 0.00528 & 0.000080 \\
\hline 0.00518 & 0.000084 \\
\hline 0.00477 & 0.000083 \\
\hline 0.00416 & 0.000079 \\
\hline 0.00344 & 0.000073 \\
\hline 0.00276 & 0.000079 \\
\hline
\end{tabular}




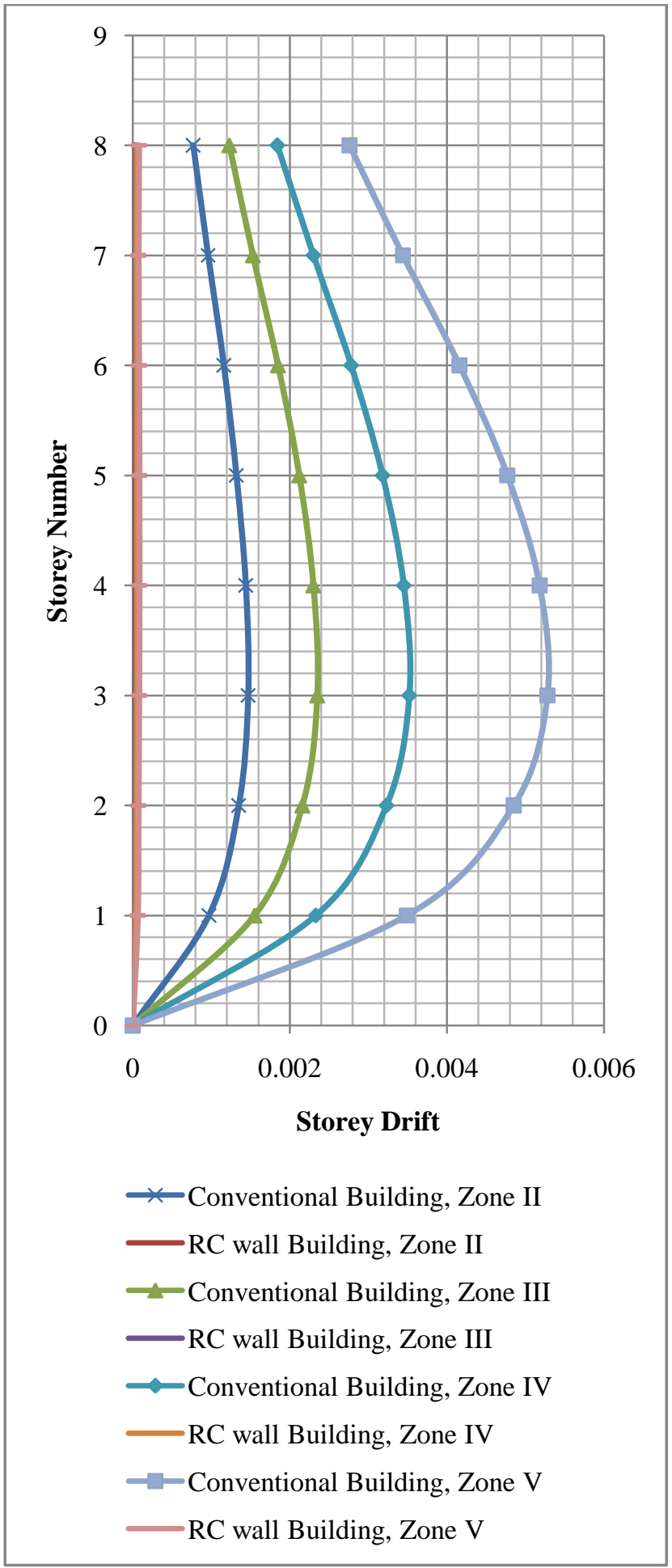

Fig-7: Storey Drift, Conventional v/s RC Wall Building, Zone II, III, IV, V (Y-direction)

As seen in the previous section in case of displacements, here it can be seen from the tables and the plots that the storey drift in the RC wall building is very much less compared to the conventional building. This result shows that the RC Wall building is safer against drift caused by the seismic forces as it produces less storey drift compared that of the conventional building in the same seismic zone.

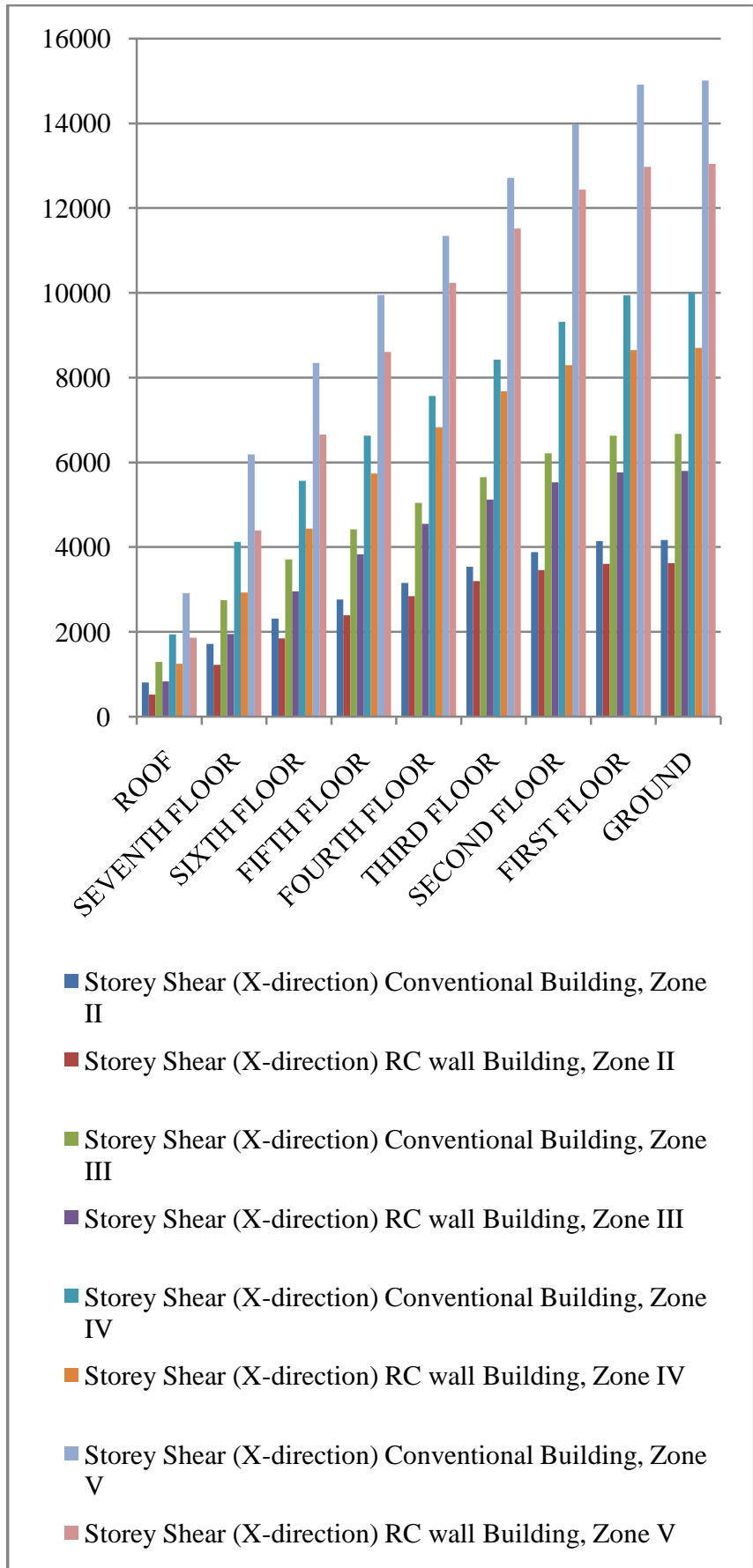

Fig-8: Storey Shear in kN v/s Storey height in X-direction

Table-17: Base Shear, Conventional v/s RC Wall Building

\begin{tabular}{|l|l|l|}
\hline \multicolumn{2}{|l|}{ Base Shear in kN } \\
\hline Zone & Conventional Building & RC Wall Building \\
\hline Zone II & 4170 & 3625 \\
\hline Zone III & 6672 & 5799 \\
\hline Zone IV & 10009 & 8699 \\
\hline Zone V & 15013 & 13048 \\
\hline
\end{tabular}




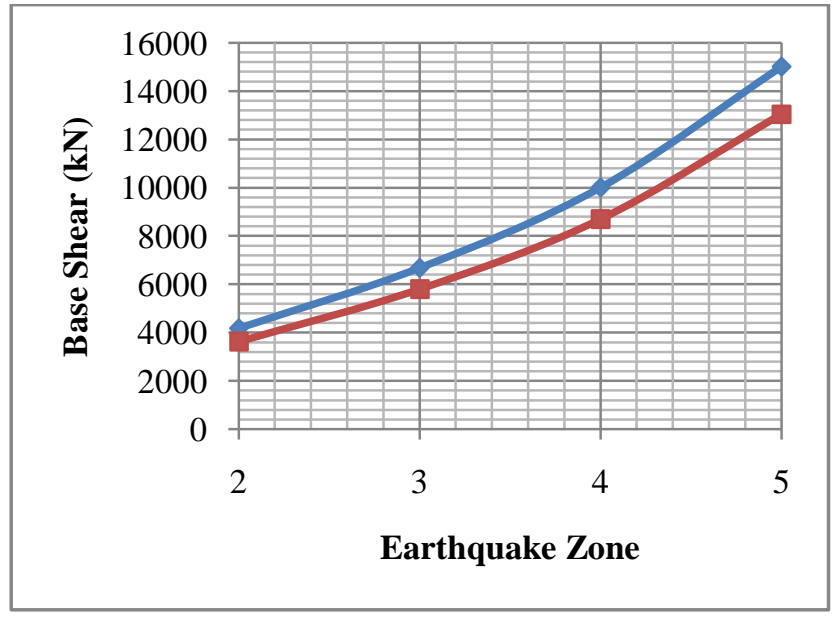

Fig-9: Base Shear, Conventional v/s RC Wall Building

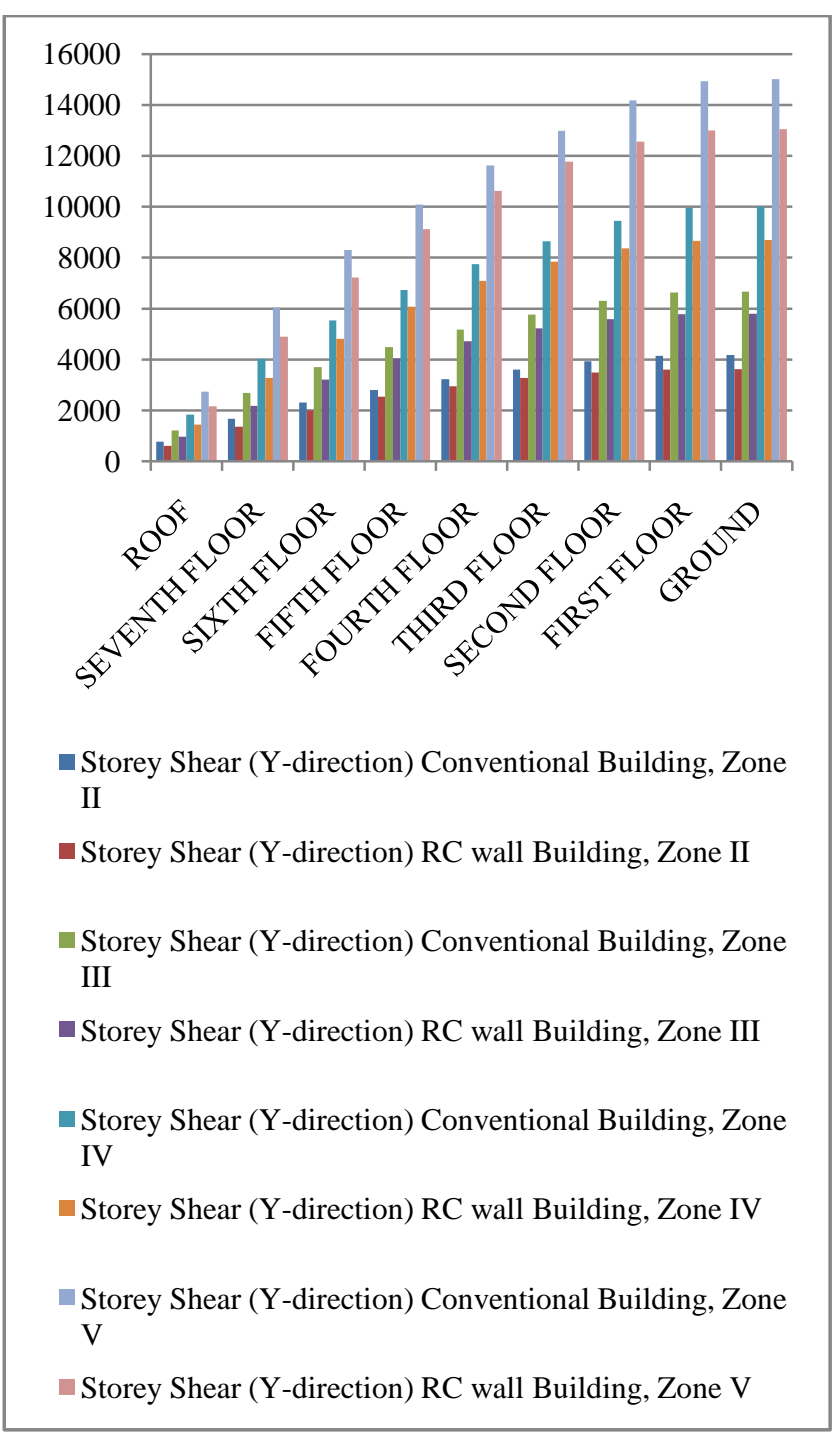

Fig-10: Storey Shear in kN v/s Storey height in Y-direction

The tables 1-8 and figures $4 \& 5$ show the variation in the storey displacements for conventional building and $\mathrm{RC}$ wall building. The tables 9-16 and figures $6 \& 7$ show the variation in the storey drift for conventional building and $\mathrm{RC}$ wall building. Table $17 \&$ figure 9 show the variation of base shear against earthquake zones.
Table-18: Modal time periods and frequencies Conventional Building v/s RC Wall Building

\begin{tabular}{|l|l|l|l|l|}
\hline \multicolumn{2}{|c|}{ Conventional Building } & \multicolumn{2}{l|}{ RC Wall Building } \\
\hline $\begin{array}{l}\text { M } \\
\text { od } \\
\text { es }\end{array}$ & $\begin{array}{l}\text { Modal } \\
\text { Time } \\
\text { Period (in } \\
\text { sec) }\end{array}$ & $\begin{array}{l}\text { Modal } \\
\text { Frequency } \\
\text { (cyc/sec) }\end{array}$ & $\begin{array}{l}\text { Modal } \\
\text { Time } \\
\text { Period (in } \\
\text { sec) }\end{array}$ & $\begin{array}{l}\text { Modal } \\
\text { Frequency } \\
\text { (cyc/sec) }\end{array}$ \\
\hline 1 & 1.418 & 0.705 & 0.165 & 6.071 \\
\hline 2 & 1.275 & 0.784 & 0.112 & 8.926 \\
\hline 3 & 1.167 & 0.857 & 0.111 & 9.017 \\
\hline 4 & 0.426 & 2.348 & 0.048 & 20.843 \\
\hline 5 & 0.374 & 2.672 & 0.037 & 27.07 \\
\hline 6 & 0.339 & 2.947 & 0.036 & 27.761 \\
\hline 7 & 0.224 & 4.454 & 0.028 & 36.179 \\
\hline 8 & 0.187 & 5.343 & 0.028 & 36.19 \\
\hline 9 & 0.168 & 5.962 & 0.025 & 40.259 \\
\hline 10 & 0.144 & 6.932 & 0.022 & 46.453 \\
\hline 11 & 0.118 & 8.453 & 0.02 & 49.088 \\
\hline 12 & 0.103 & 9.737 & 0.017 & 57.947 \\
\hline
\end{tabular}

\section{CONCLUSIONS}

From the analysis results of the fixed base buildings it can be concluded that,

1. From the tables and the plots of storey displacements it can be seen that the RC Wall Building produced much lesser displacements when considered in a particular zone and soil type. In Seismic Zone V and Soil Type II maximum displacement in Y-direction was found to be $105.65 \mathrm{~mm}$ for Conventional Building and $1.9595 \mathrm{~mm}$ for the RC Wall Building at the roof level. Thus RC Wall Building being much safer against deflections caused by earthquake.

2. It is seen from the tabulations and plots of storey drifts that the storey drift also showed much lesser values for the RC Wall Building than that of the Conventional one. This proves that the RC Wall Building is safe against the storey drifts caused by earthquake forces.

3. It can be seen from the tables and the plots that, the base shear in the RC Wall building is lesser than the conventional building. The Base Shear for Conventional Building in Zone $\mathrm{V}$ and Soil Type II was found to be $15013 \mathrm{kN}$ and for RC Wall Building it was $13048 \mathrm{kN}$.

4. It can be seen that the modal time period for $\mathrm{RC}$ Wall Building is lesser than the Conventional Building.

\section{REFERENCES}

[1]. S D Darshale and N L Shelke, Seismic Response Control of Vertically Irregular R.C.C. Structure using Base Isolation, International Journal of Engineering Research, Volume No.5 Issue: Special 2, 27-28 February 2016. 
[2]. Mahesh Kumar C L, Shwetha K G, Amrutharani H R and Jagadeesh B N, Sensitivity Analysis of LRB Isolator for RC Frame, International Journal of Science Technology \& Engineering, Volume 2, Issue 1, July 2015.

[3]. Manoj U Deosarkar and S D Gowardhan, Non Linear Dynamic Response of Combined Isolation System on Symmetric and Asymmetric Buildings, International Journal of Informative \& Futuristic Research, Volume 3, November 2015.

[4]. Vinodkumar Parma and G S Hiremath, Effect of Base Isolation in Multistoried RC Irregular Building using Time History Analysis, International Journal of Research in Engineering and Technology, Volume 4, Issue 6, June-2015.

[5]. Suhail V M and Syed Ahamed R, Seismic Analysis of R.C Buildings Provided with Hdrb Isolators of Various Rubber Thickness, International Journal of Advanced Technology in Engineering and Science, Volume No.03, Issue No. 06, June 2015.

[6]. Anusha R Reddy and Dr. V Ramesh, Seismic Analysis of Base Isolated Building in RC Framed Structures, International Journal of Civil and Structural Engineering Research, Volume 3, Issue 1, April 2015.

[7]. Vindhya Bhagavan, G V Sowjanya, Chethan Kumar B and Sandeep Kumar D S, Seismic Performance of Friction Pendulum Bearing by Considering Storey Drift and Lateral Displacement, International Journal of Research in Engineering and Technology, Volume 3, August 2014.

[8]. Khante S N and Lavkesh R Wankhade, Study of seismic response of symmetric and asymmetric base isolated building with mass asymmetry in plan, International Journal of Civil and Structural Engineering Volume X, No X, 2010.

[9]. N Torunbalci and G Ozpalanlar, Earthquake Response Analysis of Mid-Story Buildings Isolated with Various Seismic Isolation Techniques, The $14^{\text {th }}$ World Conference on Earthquake Engineering, Beijing, China, October 12-17, 2008.

[10]. Sarvesh K Jain and Shashi K Thakkar, Application of Base Isolation for Flexible Buildings, $13^{\text {th }}$ World Conference on Earthquake Engineering Vancouver, B C., Canada, August 1-6, 2004.

\section{BIOGRAPHIES}

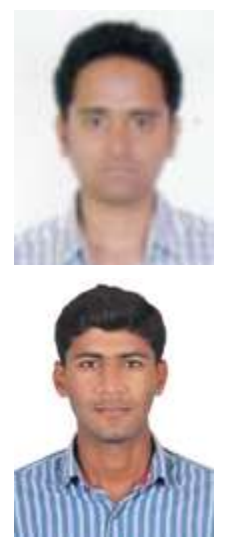

\section{Pradeep B}

M.Tech Student

Department of Civil Engineering

Global Academy of Technology,

Bengaluru-560098.

\section{Shiva Kumar KS}

Assistant Professor

Department of Civil Engineering

Global Academy of Technology,

Bengaluru-560098. 